\title{
New families of interpolating type IIB backgrounds
}

\author{
Ruben Minasian, ${ }^{a}$ Michela Petrini $^{b}$ and Alberto Zaffaroni ${ }^{c}$ \\ ${ }^{a}$ Institut de Physique Théorique, CEA/Saclay, \\ 91191 Gif-sur-Yvette Cedex, France \\ ${ }^{b}$ LPTHE, Université Paris VI, \\ 4 place Jussieu, 75252 Paris, France \\ ${ }^{c}$ Università di Milano Bicocca and INFN, sezione di Milano-Bicocca, \\ piazza della Scienza 3, Milano 20126, Italy \\ E-mail: ruben.minasian@cea.fr, petrini@lpthe.jussieu.fr, \\ Alberto.Zaffaroni@mib.infn.it
}

ABSTRACT: We construct new families of interpolating two-parameter solutions of type IIB supergravity. These correspond to D3-D5 systems on non-compact six-dimensional manifolds which are $\mathbb{T}^{2}$ fibrations over Eguchi-Hanson and multi-center Taub-NUT spaces, respectively. One end of the interpolation corresponds to a solution with only D5 branes and vanishing NS three-form flux. A topology changing transition occurs at the other end, where the internal space becomes a direct product of the four-dimensional surface and the two-torus and the complexified NS-RR three-form flux becomes imaginary self-dual. Depending on the choice of the connections on the torus fibre, the interpolating family has either $\mathcal{N}=2$ or $\mathcal{N}=1$ supersymmetry. In the $\mathcal{N}=2$ case it can be shown that the solutions are regular.

KEYWords: Flux compactifications, Gauge-gravity correspondence, String Duality

ARXIV EPRINT: 0907.5147 


\section{Contents}

1 Introduction $\quad 1$

1.1 Torsional geometry 3

1.2 The supersymmetry equations 5

$\begin{array}{lll}2 & \text { D5-brane solutions (type C) } & 7\end{array}$

2.1 Multi-center Taub-NUT solutions 8

2.2 The Eguchi-Hanson solution 9

$\begin{array}{ll}2.3 \text { Breaking supersymmetry to } \mathcal{N}=1 & 11\end{array}$

$3 \quad$ T-duality and D3-brane solutions (type B) 12

4 An interpolating solution: D5-D3 solutions 14

$\begin{array}{ll}4.1 \text { The chain of "external" dualities } & 16\end{array}$

$\begin{array}{ll}4.2 \text { The "internal" dualities } & 17\end{array}$

$\begin{array}{ll}4.3 \text { Properties of the interpolating solution } & 18\end{array}$

5 Discussion and conclusions $\quad 21$

\section{Introduction}

In recent years a lot of attention has been devoted to the study of supergravity backgrounds with non trivial fluxes. Backgrounds of this kind are indeed relevant for both string compactifications to four dimensions and the study of the gauge/gravity duality. In all cases, the geometries of interest are of warped product type: a product of four dimensional Minkowski (or Anti-de Sitter) space and an internal six-dimensional manifold, either compact or not.

A particular class of flux backgrounds is provided by solutions where the internal geometry is given by a so-called $\mathrm{SU}(3)$ structure manifold. SU(3) structure manifolds are the simplest generalisation of Calabi-Yau's, in that they also possess a globally defined holomorphic three-form and a globally defined fundamental form, but these, contrary to Calabi-Yau's, are not closed. The non closure of such forms is a measure of the backreaction of the non trivial RR and/or NS fluxes.

In the formalism of generalized complex geometry it easy to observe some general features of type IIB backgrounds with SU(3) structure: the internal manifold must be complex and there is an intricate cancellation among $\mathrm{d} J$, the exterior derivative of the fundamental form compatible with the integrable complex structure, and the NS and RR there fluxes, $H$ and $F_{3}[1,2]$.

One very special case is when the symplectic form is closed. Then the internal manifold is conformally Calabi-Yau, and the three-form fluxes form an imaginary self-dual complex 
combination. This situation is typically referred to as type B solution [3]. Another special point, which is referred as type $\mathrm{C}$, is reached when $H=0$. The only non trivial flux is the $\mathrm{RR}$ three-form, which is proportional to the exterior derivative of the fundamental form. The dilaton is running and proportional to the warp factor $(\phi=2 A)$. In a general solution, which is referred as of interpolating type, the symplectic structure is not integrable. Taking in addition a constant axion, one can show that the NS three-form flux is exact and hence trivial in cohomology.

The best known examples of gravity duals to $\mathcal{N}=1$ supersymmetric gauge theories, the Klebanov-Strassler (KS) [4] (type B) and the Maldacena-Nunez (MN) [5] (type C) solutions, fall in the SU(3) structure class of backgrounds. So does the interpolating family found in [6], which describes both the gravity dual to the baryonic branch of the KlebanovStrassler solution and an interpolation between KS and MN.

Recently a class of torsional non-Kähler geometries satisfying the conditions of $\mathcal{N}=1$ supersymmetry in purely NS backgrounds has been constructed in [7], as a subclass of principal holomorphic $\mathbb{T}^{2}$ fibrations over a four-dimensional hyper-Kähler surface $\mathcal{B}{ }^{1}$ A generic feature of such backgrounds is that they admit a balanced metric, i.e. have a fundamental form whose square is closed. In this paper, we shall use the same kinds of torsional geometry to construct type IIB backgrounds. Since we are interested in noncompact backgrounds, we shall work with non-compact base manifolds, $\mathcal{B}$, and consider multi-center Taub-NUT surfaces; the two-center case, the Eguchi-Hanson space, will be treated in a greater detail. The construction of [7] is local, and one has to ensure that the Bianchi identities are satisfied. For the holomorphic $\mathbb{T}^{2}$ fibrations over Eguchi-Hanson space, the solution of the Bianchi identity has been given in [8] for the heterotic string. For this special case, we shall heavily rely on the analysis there.

In type IIB we find a web of $\mathcal{N}=2$ backgrounds connected by different types of dualities and we discuss possible generalisations to $\mathcal{N}=1$. The simplest solution is of type $\mathrm{C}$ and describes D5-branes wrapping a two-cycle in the internal six-manifold. Due to the smooth $\mathbb{T}^{2}$ action on the internal, the background has isometries and hence can be T-dualized. The result is a type B solution, with constant dilaton (and axion), where the internal space is a direct product $\mathcal{B} \times \mathbb{T}^{2}$ but the $B$ field is not vanishing, and $H$ is non-trivial in cohomology. There is also a non-zero three-form, which is dual to the NS flux.

These two solutions are the respective counterparts of the MN and KS backgrounds. It is then natural to look for an interpolation connecting the two, in an analogy to the gravity dual to the baryonic branch [6]. Recently, a method has been proposed, [9], that allows, given any type $\mathrm{C}$ solution, to build a new one of interpolating type. The construction of [9] was motivated by a chain of dualities including an M-theory boost and was applied to reinterpret the interpolating solution of [6]. An interesting feature of the construction is that all the dualities are "external"; they do not change the structure of the internal

\footnotetext{
${ }^{1}$ Incidentally, the type $\mathrm{C}$ solution is $\mathrm{S}$-dual to the third special point, type A, where $F_{3}=0$, and which is hence pure NS type. Since the supersymmetry conditions are local, forgetting about the tadpole, the same set of requirement on the internal space would apply to purely NS background both in type II theories and in heterotic strings. In fact the local construction of the internal six-manifold that we use here [7], as well as one of the explicit examples [8] appeared originally in the heterotic context.
} 
manifold and simply modify the relation between the dilaton and the warp factor,

$$
e^{-(2 A-\phi)} \sim \sin w
$$

to include the interpolating function $w$. In view of the fact that there is no topology change between the type $\mathrm{C}$ and the interpolation, this construction is very natural. As we shall see all Bianchi identities in the general interpolating solution reduce to the type C Bianchi identity. The interpolating solutions are in fact a two parameter family of solutions, where the parameters are the M-theory boost and the asymptotic value of the dilaton. Notice that the existence of (at least) two parameters is another generic feature of type IIB interpolating families (with fixed axion).

Since our background has isometries, we can obtain the interpolating solution also using "internal" dualities. We shall show that deforming a type B solution on $\mathcal{B} \times \mathbb{T}^{2}$ by adding a closed $B$ field and then performing T-duality, we can again recover the same interpolation.

Finally, since the solutions are non compact, it would be interesting to see whether they admit a holographic gauge dual and how this looks like. A natural candidate would be a four-dimensional $\mathcal{N}=2$ gauge theory obtained by compactifying on the two torus the little string theory dual to D5-branes transverse to the ALE space. However we will not explore this in details in this paper.

The plan of the paper is as follows. Before moving to the discussion of the various solutions, we conclude the introduction with a brief review of six-dimensional internal geometry and the supersymmetry equations cast in the pure spinor form [2]. In sections 2 and 3 we describe the type $\mathrm{C}$ and its T-dual type B solutions, respectively. Then in section 4 we present the interpolating solution and discuss its properties. We briefly comment about a possible holographic interpretation in the Conclusion.

\subsection{Torsional geometry}

We are interested in supersymmetric solutions of type IIB supergravity of warped type

$$
\mathrm{d} s^{2}=e^{2 A} \mathrm{~d} s_{4}^{2}+\mathrm{d} s_{6}^{2},
$$

where the internal manifold is a six-dimensional complex non-Kähler manifold with SU(3) structure. As discussed in [7], manifolds of this kind can be obtained via $\mathbb{T}^{2}$ fibrations over a four-dimensional hyper-Kähler manifold, $\mathbb{T}^{2} \rightarrow X \stackrel{\pi}{\rightarrow} \mathcal{B}$.

Provided that the $\mathbb{T}^{2}$ bundle is non-trivial and that its curvature has no components in $\Lambda^{0,2} T^{*} \mathcal{B}$, the space of fibrations will admit a closed holomorphic three-form, and hence an integrable complex structure. It will not admit a Kähler metric. Typically, a necessary condition for preserving supersymmetry is that the internal six-manifold admits a balanced metric, i.e. has a fundamental form whose square is closed. It is satisfied by requiring that in addition, the curvature of the $\mathbb{T}^{2}$ bundle is orthogonal to the Kähler form on $\mathcal{B}$. Finally, if the curvature has no components in $\Lambda^{2,0} T^{*} \mathcal{B}$, i.e. lies completely the space of anti-self-dual two-form on the base $\Lambda^{1,1-} T^{*} \mathcal{B}$, the supersymmetry will be enhanced to $\mathcal{N}=2$.

For the metric in the base space, $\mathcal{B}$, we take

$$
\mathrm{d} s_{\mathcal{B}}^{2}=V\left(\mathrm{~d} y_{1}^{2}+\mathrm{d} y_{2}^{2}+\mathrm{d} y_{3}^{3}\right)+V^{-1}(\mathrm{~d} \tau+A)^{2},
$$


where $V$ is a harmonic function and $A$ is a connection one-form $A=A_{i} \mathrm{~d} y^{i}$. In order for the space to be hyper-Kähler, $V$ has to satisfy the monopole condition

$$
\partial_{i} V=-\epsilon_{i j k} \partial_{j} A_{k} \quad i=1,2,3 .
$$

Indeed, choosing vielbein $e^{0}=V^{-\frac{1}{2}}(\mathrm{~d} \tau+A)$ and $e^{i}=V^{\frac{1}{2}} \mathrm{~d} y^{i}(i=1,2,3)$, it is not hard to see that the two-forms

$$
\Omega_{ \pm}^{i}=e^{0} \wedge e^{i} \pm \frac{1}{2} \epsilon_{j k}^{i} e^{j} \wedge e^{k}
$$

are respectively self- and anti-self-dual $\left(* \Omega_{ \pm}^{i}= \pm \Omega_{ \pm}^{i}\right)$. Moreover the self-dual forms are closed:

$$
\mathrm{d} \Omega_{+}^{i}=0 .
$$

The forms $\Omega_{+}^{i}$ form the basis for the symplectic form $J_{\mathcal{B}}$ and the holomorphic two-form $\omega_{\mathcal{B}}$ on our four-dimensional space $[10] .^{2}$

We write the metric on the six-dimensional fibration $X$, as

$$
\mathrm{d} s_{6}^{2}=e^{2 \Delta}\left(e^{-2 u} \mathrm{~d} s_{\mathcal{B}}^{2}+\Theta \otimes \bar{\Theta}\right),
$$

where $\Theta^{I}=\mathrm{d} \theta^{I}+B^{I}(I=1,2)$ are smooth connection one-forms and we introduced the complex one-form $\Theta=\Theta^{1}+i \Theta^{2}$. The connections $\Theta^{I}$ have anti-self-dual curvature $F^{I} \in H^{2}(\mathcal{B}, \mathbb{Z}):$

$$
\pi^{*} F^{I}=\mathrm{d} \Theta^{I}, \quad * F^{I}=-F^{I} .
$$

In the complex structure defined by $\omega_{\mathcal{B}}$, these two-forms are of $(1,1)$ type. Moreover, they are orthogonal to the self-dual two forms $\Omega_{+}^{i}$

$$
F^{I} \wedge J_{\mathcal{B}}=F^{I} \wedge \omega_{\mathcal{B}}=0 .
$$

As shown in [7], for non vanishing $F$, the six-dimensional manifold $X$ does not admit a Kähler metric. It is not hard to verify that on $X$ the fundamental form and complex three-form

$$
J=e^{2 \Delta}\left(e^{-2 u} J_{\mathcal{B}}+\frac{i}{2} \Theta \wedge \bar{\Theta}\right) \quad \text { and } \quad \Omega=e^{3 \Delta-2 u} \omega_{\mathcal{B}} \wedge \Theta
$$

define an $\mathrm{SU}(3)$ structure compatible with the metric (1.6). Since the curvature $F$ has no component in $\Lambda^{0,2} T^{*} \mathcal{B}$, the fundamental form in (1.9) obeys

$$
\begin{aligned}
\mathrm{d} J= & \mathrm{d}(2 \Delta-u) \wedge J \\
& -e^{2 \Delta}\left[\mathrm{d} u \wedge\left(e^{-2 u} J_{\mathcal{B}}-\Theta^{1} \wedge \Theta^{2}\right)+\left(\pi^{*} F^{1} \wedge \Theta^{2}-\pi^{*} F^{2} \wedge \Theta^{1}\right)\right],
\end{aligned}
$$

where the second piece corresponds to the primitive part of $\mathrm{d} J$, and it is not hard to check that its wedge product with both $J$ and $\Omega$ vanishes. Similarly, on can check that the holomorphic three-form is conformally closed

$$
\mathrm{d}\left(e^{2 u-3 \Delta} \Omega\right)=0 .
$$

\footnotetext{
${ }^{2}$ Our conventions differ from those of [10], where the trio of forms defining the hyper-Kähler structure is taken to be anti-self-dual while the normalizable $(1,1)$-forms are self-dual.
} 
As it is clear from the equations above, the six-dimensional manifold $X$ is complex but is not symplectic. Moreover, wedging (1.10) with $J$, we obtain

$$
J \wedge \mathrm{d} J=\mathrm{d}(2 \Delta-u) \wedge J^{2}
$$

which tells that the metrics considered here are (conformally) balanced.

\subsection{The supersymmetry equations}

A convenient way to repackage the supersymmetry conditions in type II supergravity is in terms of pure spinors on the internal manifold. These are polyforms, obtained as tensor products of the supersymmetry parameters on the internal manifold

$$
\Psi_{ \pm}=\frac{8}{|a|^{2}} e^{-\phi} e^{-B} \eta_{+}^{1} \oplus \eta_{ \pm}^{2 \dagger}
$$

where $|a|^{2}=\left\|\eta_{+}^{1}\right\|^{2}=\left\|\eta_{+}^{1}\right\|^{2}$. Then the supersymmetry equations become $[1,2]$

$$
\begin{aligned}
\mathrm{d}\left(e^{3 A} \Psi_{-}\right) & =0, \\
\mathrm{~d}\left(e^{2 A} \operatorname{Re} \Psi_{+}\right) & =0, \\
\mathrm{~d}\left(e^{4 A} \operatorname{Im} \Psi_{+}\right) & =e^{4 A} e^{-B} *\left(F_{1}-F_{3}+F_{5}\right),
\end{aligned}
$$

where $\phi$ is the dilaton, $B$ is the NS two-form and $F_{1}, F_{3}, F_{5}$ the RR fluxes on the internal manifolds. In these equations we have already used the fact that supersymmetry sets the norm of the spinors to be proportional to the warp factor, $|a|^{2}=e^{A}$. So we see that supersymmetry requires that one of the pure spinors must be closed, while the second one is not integrable due to the presence of the $\mathrm{RR}$ fluxes.

For an $\mathrm{SU}(3)$ structure manifold the pure spinors $\Psi_{ \pm}$are given by

$$
\Psi_{+}=e^{i \theta_{+}} e^{-\phi} e^{-B} e^{-i J} \quad \text { and } \quad \Psi_{-}=-i e^{i \theta_{-}} e^{-\phi} e^{-B} \Omega
$$

where $J$ and $\Omega$ are the fundamental form and the holomorphic three-form defining the $\mathrm{SU}(3)$ structure. The choice of one phase is arbitrary. ${ }^{3}$ In this paper we fix them in such a way to be consistent with the interpolation solution of $[6]$

$$
\theta_{+}=w+\frac{\pi}{2} \quad \theta_{-}=-\frac{\pi}{2}
$$

where $w$ is the interpolating function.

\footnotetext{
${ }^{3}$ The SU(3) structure is equivalently defined by a globally defined, nowhere vanishing chiral spinor on the internal manifold. If we call such a spinor $\eta_{+}$, then the two supersymmetry parameters are related to $\eta_{+}$by

$$
\eta_{+}^{1}=e^{A / 2} e^{i \theta_{a}} \eta_{+} \quad \eta_{+}^{2}=e^{A / 2} e^{i \theta_{b}} \eta_{+}
$$

and the phases of the pure spinors are given by $\theta_{ \pm}=\theta_{a} \mp \theta_{b}$.
} 
Expanding equations (1.14a) and (1.14b) into forms of definite degree yields the following equations for the NS sector

$$
\begin{aligned}
\sin w \mathrm{~d}(2 A-\phi) & =-\mathrm{d}(\sin w), \\
\cos w e^{-2 A} \mathrm{~d}\left(e^{2 A} J\right)+e^{\phi} \mathrm{d}\left(e^{-\phi} \cos w\right) J & =-\sin w H, \\
\sin w J \wedge \mathrm{d} J & =\cos w H \wedge J, \\
\mathrm{~d}\left(e^{3 A-\phi} \Omega\right) & =0, \\
\Omega \wedge H & =0,
\end{aligned}
$$

and expanding (1.14c) yields for the RR fluxes

$$
\begin{aligned}
& * F_{1} e^{\phi}=e^{\phi} \mathrm{d}\left(e^{-\phi} \cos w\right) \wedge J^{2} / 2 \\
& * F_{3} e^{\phi}=-\sin w e^{-2 A} \mathrm{~d}\left(e^{2 A} J\right)+\cos w H \\
& * F_{5} e^{\phi}=e^{-4 A+\phi} \mathrm{d}\left(e^{4 A-\phi} \cos w\right)
\end{aligned}
$$

Equation (1.18d) comes for the closure of $\Psi_{-}$, and indicates that the complex structure is integrable. This allows to use the Dolbeault operator in taking the Hodge star, so that the RR fluxes can be written as

$$
\begin{aligned}
& F_{1}=i(\partial-\bar{\partial})\left(\cos w e^{-\phi}\right) \quad \Rightarrow \quad \bar{\partial}\left(C+i \cos w e^{-\phi}\right)=0, \\
& F_{5}=i e^{-4 A}(\partial-\bar{\partial})\left(\cos w e^{4 A-\phi}\right) \wedge J^{2} / 2
\end{aligned}
$$

where $C$ is the RR zero form potential. So the presence of a holomorphic combination of the axion and the dilaton is a generic feature of all these backgrounds. In this paper we shall be interested in the solutions where the axion is constant.

The expression for the RR three-form (1.19b) can be simplified as well, but the result depends on the value of $w$. For $w=\pi$, it reduces to

$$
e^{\phi} F_{3}=* H
$$

When $w \neq \pi$, it is not hard to show that the two form $e^{(2 A-\phi)} J$ is primitive. This allows to simplify the expression for RR three-form flux to

$$
F_{3}=-i \sin w\left(e^{-2 A}(\partial-\bar{\partial})\left(e^{2 A-\phi} J\right)-e^{-2 \phi} J \wedge(\partial-\bar{\partial}) e^{\phi}\right)-e^{-\phi} \cos w * H .
$$

Note that in our conventions, the $(2,1)$ primitive form is imaginary self-dual.

For generic values of the phase $w$ the system of equations (1.18a)-(1.19c) describes a solution of an "interpolating" type. The special cases of $w=\pi(\bmod \pi)$ and $w=\pi / 2(\bmod \pi)$ are often referred to as type $\mathrm{B}$ and type $\mathrm{C}$ solutions in the literature. Type $\mathrm{C}$ solutions are characterised by a vanishing axion and NS three-form, $C=0$ and $H=0$. On the contrary, type B solutions have an holomorphic dilaton (1.20a) which reduces to the standard complex field $\tau=C+i e^{-\phi}$, and we recover the imaginary self-dual three-form $F_{3}+\tau H$. Moreover $H \wedge J=0$ and $J$ is conformally closed. 
As always, the supersymmetry equations are only necessary condition for supersymmetric vacua. In order for a background to be a solution, it must also satisfy the Bianchi identities for the fluxes (the equations of motion are implied by supersymmetry)

$$
\begin{aligned}
& \mathrm{d} F_{1}=0, \\
& \mathrm{~d} F_{3}-H \wedge F_{1}=0, \quad \mathrm{~d} H=0, \\
& \mathrm{~d} F_{5}-H \wedge F_{3}=0 \text {. }
\end{aligned}
$$

\section{D5-brane solutions (type C)}

In this section we discuss solutions of type C, namely with $w=\pi / 2$. A generic feature of this class of solutions is that the internal manifold is complex but not Kähler.

For $\cos w=0$, the ansatz (1.9) combined with (1.18a), (1.18c) and (1.18d) gives

$$
2 A=2 \Delta=\phi=u .
$$

In order to simplify notations, here and in the following, we set most of the integration constants to one. They can be easily reintroduced as constant parameters multiplying the various terms in the metric.

As for the other equations, (1.18b), (1.19a) and (1.19c) imply

$$
H=0 \quad F_{1}=F_{5}=0,
$$

with the only non-vanishing flux given by (1.19b)

$$
\mathrm{d}\left(e^{2 A} J\right)=-e^{4 A} * F_{3} .
$$

Using the integrability of the complex structure (1.18d), it is easily checked that

$$
F_{3}=i(\partial-\bar{\partial})\left(e^{-2 A} J\right) .
$$

Let us consider type $\mathrm{C}$ solutions where the internal manifolds are torus fibrations of the type discussed in section 1.1. The internal metric is then

$$
\mathrm{d} s_{6 C}^{2}=e^{-\phi} \mathrm{d} s_{\mathcal{B}}^{2}+e^{\phi} \Theta \otimes \bar{\Theta},
$$

and the tadpole condition reads

$$
\mathrm{d} F_{3}=-2 i \partial \bar{\partial}\left(e^{-2 A} J\right)=-2 i \partial \bar{\partial}\left(e^{-2 \phi} J_{\mathcal{B}}+\frac{i}{2} \Theta \wedge \bar{\Theta}\right) .
$$

Using the closure of the fundamental form on the hyper-Kähler base and the curvature of the torus fibration, the absence of tadpoles is equivalent to the following equation

$$
2 i \partial \bar{\partial} e^{-2 \phi} \wedge J_{\mathcal{B}}-F \wedge \bar{F}=0,
$$

which is the only equation left to solve. 
In solving the supersymmetry constraints we used the fundamental form and complex three-form defined in (1.9)

$$
J=e^{-\phi} J_{\mathcal{B}}+e^{\phi} \frac{i}{2} \Theta \wedge \bar{\Theta}, \quad \Omega=e^{-\phi / 2} \omega_{\mathcal{B}} \wedge \Theta .
$$

It is easy to see that, by a change of complex structure on the base $\mathcal{B}$, we obtain a second $\mathrm{SU}(3)$ structure

$$
J=-e^{-\phi} J_{\mathcal{B}}+e^{\phi} \frac{i}{2} \Theta \wedge \bar{\Theta} \quad \text { and } \quad \Omega=e^{-\phi / 2} \bar{\omega}_{\mathcal{B}} \wedge \Theta
$$

which solves all the supersymmetry conditions with the same metric and RR flux. This means that the solution preserves $\mathcal{N}=2$ supersymmetry.

\subsection{Multi-center Taub-NUT solutions}

We can find solutions of the tadpole conditions (2.7) where the base metric (1.2) is of Gibbons-Hawking type. These are obtained choosing the function $V$ in (1.2) as

$$
V=\eta+\sum_{q=1}^{N} \frac{1}{r_{q}},
$$

where the index $q$ denotes the individual centers and $r_{q}=\left|\vec{y}-\vec{y}_{q}\right|$ is the distance away from a given center, $y_{q}$. For $\eta=1$, the metric corresponds to a regular multi-center Taub-NUT . For $\eta=0$, we have the ALE spaces. For the special values $q=1$ and $q=2$, the base is flat space or an Eguchi-Hanson space, respectively.

As discussed in section 1.1 we need to construct a $\mathbb{T}^{2}$ fibration with anti-self dual $(1,1)$ curvature. This can be done using the anti-self-dual two forms $\Omega_{-}^{i}$ in (1.4). Indeed, for a choice of (three-dimensional) harmonic functions $K^{I}$ and one-form $\xi^{I}$ (we shall take $I=1,2$ ), satisfying the (anti)monopole equation [10]

$$
\partial K^{I}=+\epsilon_{i j k} \partial_{j} \xi_{k}^{I}
$$

the potentials

$$
B^{I}=V^{-\frac{1}{2}} K^{I} e^{0}+\xi^{I}
$$

will yield anti-self-dual field strengths

$$
F^{I}=\mathrm{d} B^{I}=-\partial_{i}\left(V^{-1} K^{I}\right) \Omega_{-}^{i} .
$$

Then the torus fibration is defined by $\Theta^{I}=d \theta^{I}+B^{I}$.

On a Taub-NUT space with $N$ centers there are $N-1$ normalizable anti-self dual $(1,1)$ forms corresponding to the $N-1$ independent compact two-cycles. Regularity and normalizability require that the positions of the poles of the harmonic functions $K^{I}$ coincide with the centers of the Taub-NUT. The function

$$
K=K^{1}+i K^{2}=\sum_{q=1}^{N} \frac{k_{q}}{r_{q}}
$$

defines a smooth fibration with complex curvature $F=F_{1}+i F_{2}=-\partial_{i}\left(V^{-1} K\right) \Omega_{-}^{i}$. 
The three-form in this case is

$$
F_{3}=i(\partial-\bar{\partial})\left(e^{-2 \phi}\right) \wedge J_{\mathcal{B}}+\frac{1}{2}(\Theta \wedge \bar{F}+\bar{\Theta} \wedge F),
$$

and the tadpole condition $(2.7)$

$$
2 i \partial \bar{\partial} e^{-2 \phi} \wedge J_{\mathcal{B}}-F \wedge \bar{F}=0
$$

is equivalent to

$$
\square_{\mathcal{B}}\left(e^{-2 \phi}\right)=*(F \wedge \bar{F}) .
$$

When the function $\phi$ only depends on the $y^{i}$ coordinates, (2.17) reduces to the differential equation

$$
\frac{\nabla^{2} e^{-2 \phi}}{V}+\left|\vec{\nabla} \frac{K}{V}\right|^{2}=0
$$

where derivatives are taken with respect the $y_{i}$ variables in $\mathbb{R}^{3}$. The tadpole equation can be explicitly solved by

$$
e^{-2 \phi}=-\frac{K \bar{K}}{2 V}+L
$$

with $L\left(y_{i}\right)$ an arbitrary harmonic function on $\mathbb{R}^{3}$. Since $K / V$ is smooth by definition, the first term on the right hand-side has simple poles at the positions of the Taub-NUT centers. All these singularities can be cancelled and the expression made positive by a suitable choice of coefficients in $L=l_{0}+\sum_{q} \frac{l_{q}}{r_{q}}$. The resulting metric and dilaton are everywhere regular.

These are solutions with non trivial fluxes and no branes. By allowing poles in $e^{-2 \phi}$ we could find more general solutions with $\mathrm{d} F_{3} \neq 0$ corresponding to D5-branes wrapping the two-torus.

\subsection{The Eguchi-Hanson solution}

Setting $\eta=0$ in the potential (2.10), we obtain the family of ALE spaces. It is interesting to see the explicit expression for the solution in the simplest case of ALE, the EguchiHanson space $T^{*} P_{1}(\mathbb{C})$. It corresponds to the resolution of $\mathbb{C}^{2} / \mathbb{Z}_{2}$, where the singular point is replaced by a two-sphere. A similar solution has been recently discussed in [8] in the heterotic framework.

Following [8], we use a different set of coordinates with respect to the previous section, which fully exploits the $\mathrm{SO}(3)$ invariance of the metric.

The Kähler form and holomorphic two-form on the smooth Eguchi-Hanson are given by

$$
\begin{aligned}
J_{\mathcal{B}} & =\frac{i}{2} \partial \bar{\partial} K\left(r^{2}\right)=\frac{i}{2}\left[\frac{1}{r^{2}} \sqrt{r^{4}+a^{4}} \partial \bar{\partial} r^{2}-\frac{a^{4}}{r^{4} \sqrt{r^{4}+a^{4}}} \partial r^{2} \wedge \bar{\partial} r^{2}\right], \\
\omega_{\mathcal{B}} & =\mathrm{d} z_{1} \wedge \mathrm{d} z_{2},
\end{aligned}
$$

where $r^{2}=\left|z^{1}\right|^{2}+\left|z^{2}\right|^{2}$, the Kähler potential is

$$
K\left(r^{2}\right)=\sqrt{a^{4}+r^{4}}+a^{2} \log \left(\frac{r^{2}}{a^{2}+\sqrt{a^{4}+r^{4}}}\right),
$$

and $a$ is a non-negative constant controlling the size of the two-sphere. 
For the Eguchi-Hanson space there exists a single normalizable anti-self dual closed $(1,1)$ form. It corresponds to the curvature of a line bundle on the two-sphere. The explicit expression has been constructed in [8] and it is given by $i \partial \bar{\partial} \log h$ with

$$
\frac{\mathrm{d}}{\mathrm{d} r^{2}} \log h=\frac{1}{r^{2} \sqrt{a^{4}+r^{4}}} .
$$

We can take this to be the curvature of the $\mathbb{T}^{2}$ bundle. If we introduce complex coordinates on the torus, $\theta=\theta^{1}+i \theta^{2}$, then the one-form connection is chosen

$$
\Theta=\mathrm{d} \theta-i c \partial \log h
$$

in such a way that $F=\mathrm{d} \Theta=\bar{\partial} \Theta . c$ is a quantized constant, essentially the first Chern number of the torus fibration. Then the three-form flux (2.4) reads

$$
F_{3}=\frac{1}{2}\left[-\frac{\mathrm{d}}{\mathrm{d} r^{2}}\left(e^{-2 \phi}\right) \frac{\sqrt{r^{4}+a^{4}}}{r^{2}} \partial \bar{\partial} r^{2} \wedge(\partial-\bar{\partial}) r^{2}+\Theta \wedge \bar{F}+\bar{\Theta} \wedge F\right] .
$$

As before we consider solutions corresponding to pure fluxes. In this case the tadpole condition, $\mathrm{d} F_{3}=0$, reduces to

$$
2 i \partial \bar{\partial}\left(e^{-2 \phi} J_{\mathcal{B}}+\frac{i}{2} \Theta \wedge \bar{\Theta}\right)=\frac{1}{r^{2}} \frac{\mathrm{d}}{\mathrm{d} r^{2}}\left(\mathcal{J}\left(r^{2}\right) r^{4}\right) \mathrm{d} z^{1} \wedge \mathrm{d} \bar{z}^{1} \wedge \mathrm{d} z^{2} \wedge \mathrm{d} \bar{z}^{2},
$$

where

$$
\mathcal{J}\left(r^{2}\right)=-\frac{\mathrm{d}}{\mathrm{d} r^{2}}\left(e^{-2 \phi}\right) \frac{1}{r^{2}} \sqrt{r^{4}+a^{4}}+\frac{|c|^{2}}{r^{4}\left(a^{4}+r^{4}\right)} .
$$

The general solution of the tadpole condition is $\mathcal{J}\left(r^{2}\right)=\gamma / r^{4}$, which leads to

$$
e^{-2 \phi}=\frac{|c|^{2}}{a^{4} \sqrt{a^{4}+r^{4}}}+\frac{a^{4} \gamma-|c|^{2}}{a^{6}} \log \left[\frac{2 a^{4}\left(a^{2}+\sqrt{a^{4}+r^{4}}\right)}{\left(a^{4} \gamma-|c|^{2}\right) r^{2}}\right]+e^{-2 \phi_{\infty}} .
$$

This expression is singular at $r=0$ for generic values of the constant $\gamma$. However for the special value $\gamma=|c|^{2} / a^{4}$ the solution reduces to a regular one

$$
e^{-2 \phi}=\frac{|c|^{2}}{a^{4} \sqrt{a^{4}+r^{4}}}+e^{-2 \phi_{\infty}} .
$$

Note that for generic values of $\phi_{\infty}$ the warp factor approaches a constant for large $r$. In the same limit, the curvature $F$ goes to zero and the torus fibration becomes trivial. We obtain a solution which is an asymptotic unwarped ALE space times a two torus. On the other hand, for small $r$, the non-trivial fibration changes the topology of the internal space. In particular, one of the two torus circles combines with the two-sphere in the Eguchi-Hanson space to give a non-trivial three-cycle ${ }^{4}[7]$. Thus for small $r$, topologically, the space becomes $S^{3} \times \mathbb{R}^{2} \times S^{1}$. Since $F^{1}$ and $F^{2}$ are proportional, a one-cycle remains non-trivial [7].

\footnotetext{
${ }^{4}$ In fact, the Eguchi-Hanson space becomes $\mathbb{R}^{2} \times S^{2}$ and one cycle in the two-torus is fibered over $S^{2}$ with the connection proportional to that of the Hopf fibration.
} 
The presence of $F_{3}$ suggests a brane interpretation. Indeed the solution is similar in spirit to the Maldacena-Nunez solution and its generalization [11] recently discussed in [9]. We can even take a near-brane limit by sending $e^{-2 \phi_{\infty}}$ to zero. For large $r$, the metric becomes a warped product,

$$
\mathrm{d} s^{2} \sim r\left(\mathrm{~d} s_{4}^{2}+\mathrm{d} \theta \otimes \mathrm{d} \bar{\theta}\right)+\frac{1}{r} \mathrm{~d} s_{\mathcal{B}}^{2},
$$

and the three-form flux reduces to

$$
F_{3} \sim \frac{1}{r^{4}} \partial \bar{\partial} r^{2} \wedge(\partial-\bar{\partial}) r^{2} .
$$

It is easy to check that this expression is the volume form of the three-sphere at infinity in the Eguchi-Hanson space. We thus obtain a warped solution which, asymptotically, represents the near-horizon geometry of a D5 brane wrapped on the two-torus. On the other hand, the two-torus disappears in the IR part of the solution. As in a geometric transition, the brane is replaced by a flux supported on the non-trivial three-cycle. Indeed, the first term in (2.24) vanishes and the three-form reduces to

$$
F_{3} \sim \Theta \wedge \bar{F}+\bar{\Theta} \wedge F
$$

which is indeed supported on the IR three-sphere.

It is important to notice that a non-trivial fibration is crucial for obtaining a regular solution for small $r$. A D5 brane solution in flat space or on $\mathbb{C}^{2} / Z_{2}$ is singular in the IR. Even the resolution of the orbifold to a smooth ALE space still leads to a singular solution, as can be seen from equation (2.27) in the limit $c \rightarrow 0$. However, for $c \neq 0$ we obtain a change in topology that allows for a regular solution.

The large value of the dilaton suggests that we should better use the S-dual picture representing an NS brane. For large $r$ the S-dual solution behaves as

$$
\begin{aligned}
\mathrm{d} s_{N S}^{2} & \sim \mathrm{d} s_{4}^{2}+\mathrm{d} \theta \otimes \mathrm{d} \bar{\theta}+\frac{\mathrm{d} r^{2}}{r^{2}}+\mathrm{d} s_{S^{3} / \mathbb{Z}_{2}}^{2}, \\
e^{\phi_{N S}} & \sim \frac{1}{r},
\end{aligned}
$$

which represents a linear dilaton background. This is the holographic dual of a little string theory living on a five-brane sitting at $\mathbb{C}^{2} / Z_{2}$ and compactified on a torus [12].

A similar analysis can be performed for the general solution based on Taub-NUT spaces.

\subsection{Breaking supersymmetry to $\mathcal{N}=1$}

There is a simple generalization of the solutions obtained above by adding a $(2,0)$ term to the torus curvature

$$
\mathrm{d} \Theta=F+G,
$$

where $G \in H^{(2,0)}(\mathcal{B}, \mathbb{Z})$. The total curvature $F+G$ is still orthogonal to the fundamental and holomorphic two-forms on $\mathcal{B}$

$$
(F+G) \wedge J_{\mathcal{B}}=(F+G) \wedge \omega_{\mathcal{B}}=0,
$$


and this is enough to solve equations (1.18c) and (1.18d) and all the other supersymmetry constraints. Since a reverse in the complex structure of $\mathcal{B}$ will transform a $(2,0)$ form into a $(0,2)$ one, this solution will preserve only one of the two supersymmetries.

The general form of the new piece is $G=g \omega_{\mathcal{B}}$, where $g$ is an arbitrary holomorphic function on $\mathcal{B}$, which can be non-trivial since the space is non compact. For simplicity we analyse the solution with $\mathcal{B}$ given by the Eguchi-Hanson space and $g$ constant. In this case it is easy to see what happens to the tadpole condition, which becomes

$$
\begin{aligned}
2 i \partial \bar{\partial}\left(e^{-2 \phi} J_{\mathcal{B}}+\frac{i}{2} \Theta \wedge \bar{\Theta}\right) & =2 i \partial \bar{\partial}\left(e^{-2 \phi}\right) \wedge J_{\mathcal{B}}-F \wedge \bar{F}+G \wedge \bar{G} \\
& =2 i \partial \bar{\partial}\left(e^{-2 \phi}\right) \wedge J_{\mathcal{B}}+\left(|F|^{2}+|G|^{2}\right) \mathrm{Vol}_{4},
\end{aligned}
$$

since $\bar{\partial} \Theta=F$ and $\partial \Theta=G$. Using (2.20), we see that the tadpole equation has the same form as in (2.25) with an additional constant contribution to the function $\mathcal{J}\left(r^{2}\right)$

$$
\mathcal{J}\left(r^{2}\right)=-\frac{\mathrm{d}}{\mathrm{d} r^{2}}\left(e^{-2 \phi}\right) \frac{1}{r^{2}} \sqrt{r^{4}+a^{4}}+\frac{|c|^{2}}{r^{4}\left(a^{4}+r^{4}\right)}-\frac{|g|^{2}}{2} .
$$

The regular solution of the tadpole condition $\mathcal{J}\left(r^{2}\right)=\gamma / r^{4}$ is now

$$
e^{-2 \phi}=\sqrt{a^{4}+r^{4}}\left(-\frac{|g|^{2}}{2}+\frac{c^{2}}{a^{4}\left(a^{4}+r^{4}\right)}\right)+\delta .
$$

This expression changes sign and it is not well behaved. The presence of a $(2,0)$ piece in the curvature unpleasantly changes the asymptotic behavior of the solution.

It would be of some interest to explore further the possibility of having regular $\mathcal{N}=1$ solutions.

\section{T-duality and D3-brane solutions (type B)}

Examples of type B solutions with $w=\pi$ can be obtained from the type $\mathrm{C}$ backgrounds discussed above via T-duality. In general, type B solutions can have a holomorphic dilaton. A particular class is obtained for constant dilaton and vanishing axion. The metric is conformally Calabi-Yau and the (complex) three-form flux is imaginary self-dual.

Since the original type C-solution has two isometries, corresponding to shifts in the angles $\theta^{I}$ on the torus, we can perform two T-dualities along those directions. The effect of T-duality is to untwist the torus fibration and to produce a $B$ field with one leg along the torus. The type $\mathrm{B}$ dilaton is constant while the original type $\mathrm{C}$ dilaton is related to the type B warp factor. Indeed, a standard application of the T-duality rules gives

$$
\begin{aligned}
\mathrm{d} s_{6}^{2} & =e^{-2 A}\left(\mathrm{~d} s_{\mathcal{B}}^{2}+\mathrm{d} \theta \otimes \mathrm{d} \bar{\theta}\right), \\
e^{\phi} & =g_{s} \\
A & =\phi_{C} / 2 \\
B & =B^{1} \wedge \mathrm{d} \theta^{1}+B^{2} \wedge \mathrm{d} \theta^{2},
\end{aligned}
$$

where $B^{I}$ are the potentials defined in $(2.12)$ and the function $\phi_{C}$ denotes the dilaton of the type $\mathrm{C}$ solution. This was determined as the solution of the tadpole equation of 


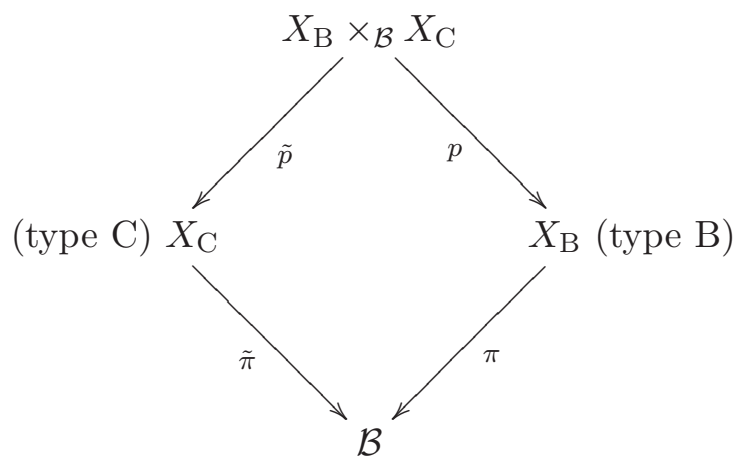

Figure 1. The solutions B and C are related via standard T-duality and can be obtained respectively via projections $\tilde{p}$ and $p$ form the correspondence space. $\tilde{\pi}$ and $\pi$ are the projections of dual torus bundles to base $\mathcal{B}$.

type C, (2.19) for the general Taub-NUT solutions and (2.28) for the Eguchi-Hanson case. Notice that in type B the warp factor satisfies the equation

$$
\square_{\mathcal{B}}\left(e^{-4 A}\right)=*(F \wedge \bar{F}) .
$$

The result of T-duality can be summarized in the standard picture of the correspondence space [13] (see figure 1). On one side, we have a non trivial fibration

$$
\mathbb{T}^{2} \rightarrow X_{\mathrm{C}} \stackrel{\pi}{\rightarrow} \mathcal{B}
$$

with a vanishing $H$ (which is parametrized by a trivial two-torus). On the other, the manifold is a direct product

$$
X_{\mathrm{B}}=\mathcal{B} \times \mathbb{T}^{2}
$$

and the geometrisation of the non trivial $H$ yields another nontrivial two-torus fibration over the base $\mathcal{B}$. The correspondence space is a fiberwise product $X_{\mathrm{B}} \times_{\mathcal{B}} X_{\mathrm{C}}$, and is a double $\mathbb{T}^{2}$ fibration over $\mathcal{B}$ with one of the fibrations being trivial and the the other having a connection with curvature $F$. The roles of these two tori are reversed in going from type B solution to type C.

The RR fluxes for the T-dual solution can be obtained from those of type $\mathrm{C}$ by

$$
\left.e^{-B} F\right|_{\text {typeB }}=\left.\left(\iota_{\theta^{1}}+\mathrm{d} \theta^{1}\right) \cdot\left(\iota_{\theta^{2}}+\mathrm{d} \theta^{2}\right)\left(e^{-B} F\right)\right|_{\text {typeC }},
$$

where $F=F_{1}+F_{3}+F_{5}$ is the sum of the RR field strength on the internal manifold. In this case we obtain

$$
\begin{aligned}
F_{1} & =0 \\
g_{s} F_{3} & =-F^{1} \wedge \mathrm{d} \theta^{2}+F^{2} \wedge \mathrm{d} \theta^{1}, \\
g_{s} F_{5} & =i(\partial-\bar{\partial})\left(e^{-4 A}\right) \wedge J_{\mathcal{B}} \wedge \mathrm{d} \theta^{1} \wedge \mathrm{d} \theta^{2},
\end{aligned}
$$

where $g_{s}$ is the constant value of the dilaton. 
From (3.5) one sees that the Bianchi identities for the RR fields of the type B solution are automatically satisfied if the type $\mathrm{C}$ ones are. ${ }^{5}$

The solution has an interpretation in terms of D3-branes. Let us discuss for simplicity the case of the Eguchi-Hanson solution. In the near brane limit, $e^{-2 \phi_{\infty}} \rightarrow 0$, the metric asymptotes at large $r$

$$
\mathrm{d} s^{2} \sim r \mathrm{~d} s_{4}^{2}+\frac{1}{r}\left(\mathrm{~d} \theta \otimes \mathrm{d} \bar{\theta}+\mathrm{d} s_{\mathcal{B}}^{2}\right)
$$

corresponding to D3-branes smeared on the two-torus. The metric remains a trivial product of the ALE space with a torus also at smaller values of $r$. On the other hand, we have an imaginary self dual flux for the three-forms. Notice that the $F_{5}$ flux vanishes for small values of $r$. Solutions of this type have been discussed in [15], in the case where the two-torus is replaced by $\mathbb{R}^{2}$, and have been interpreted as a combination of physical and fractional D3 branes probing a resolved $\mathbb{C}^{2} / Z_{2}$ singularity. The vanishing of physical D3 brane charge in the IR is typical for these kinds of solution. The solutions of [15] are singular in the IR. We see that, in the case where we compactify two directions, it is possible to find a regular solution.

Finally let us discuss the T-duality transformation on pure spinors. The type $\mathrm{C}$ solution has a trivial $B$-field, and thus the generalized tangent bundle is simply given by $T X_{\mathrm{C}} \oplus$ $T^{*} X_{\mathrm{C}}$. The pure spinors are globally defined and using (2.1) can be written as

$$
\begin{aligned}
& \left.\Psi_{+}\right|_{\text {type } \mathrm{C}}=-e^{-\phi_{C}} \exp \left[-i\left(e^{-\phi_{C}} J_{\mathcal{B}}+\frac{i}{2} e^{\phi_{C}} \Theta \wedge \bar{\Theta}\right)\right], \\
& \left.\Psi_{-}\right|_{\text {type } C}=-e^{-3 \phi_{C} / 2} \omega_{\mathcal{B}} \wedge \Theta .
\end{aligned}
$$

Note that since the axion is vanishing in this solution, only four-manifolds with globally defined $\omega_{\mathcal{B}}$, namely the hyper-Kähler ones, can be admissible bases. Since the pure spinors are also polyforms, T-duality act on them as in (3.5). Then T-duality along the two-torus maps (3.8) into a pair of type B pure spinors:

$$
\begin{aligned}
& \left.\Psi_{+}\right|_{\text {type } \mathrm{B}}=-i e^{-B-i J}, \\
& \left.\Psi_{-}\right|_{\text {type } \mathrm{B}}=-e^{-B} \wedge \Omega,
\end{aligned}
$$

with the fundamental form and the holomorphic three-form given by

$$
\begin{aligned}
& J=e^{-2 A}\left(J_{\mathcal{B}}+\mathrm{d} \theta^{1} \wedge \mathrm{d} \theta^{2}\right), \\
& \Omega=i e^{-3 A} \omega_{\mathcal{B}} \wedge\left(\mathrm{d} \theta^{1}+i \mathrm{~d} \theta^{2}\right),
\end{aligned}
$$

since $A=\phi_{C} / 2$.

\section{An interpolating solution: D5-D3 solutions}

While finding solutions of $\mathrm{B}$ and $\mathrm{C}$ type is relatively simple, solutions corresponding to generic values of the parameter $w$ are harder to find. A general discussion of the features

\footnotetext{
${ }^{5}$ Bianchi identities $\mathrm{d}\left(e^{-B} F\right)=0$ are automatically satisfied when a shift in $\theta^{i}$ is an isometry $\left(\mathcal{L}_{\theta^{i}}\left(e^{-B} F\right)=0\right)$ since $\mathrm{d}$ and the operators $\iota_{\theta^{i}}+\mathrm{d} \theta^{i}$ anticommute. For the T-duality conventions we refer to [14].
} 
of interpolating solutions was given in [16]. A notable regular example is provided by the solution [6] describing the baryonic branch of the Klebanov-Strassler solution, where the interpolation parameter is interpreted as the VEV of a baryonic operator in the dual field theory. The solution interpolates between Klebanov-Strassler (type B) and MaladacenaNuñez (type C), when the parameters corresponding to the baryonic VEV and the gauge coupling constant are judiciously varied. Another interesting (although singular) example is given by the gravity dual of non commutative D5 branes, where the interpolation parameter is given by the value of a $B$ field $[17,18]$. Here we present an interpolating solution which is regular and combines features of both these examples. The solution can be obtained in two different ways

- starting from point $\mathrm{C}$, we can apply the prescription of [9], which, through a chain of dualities, generates a family of interpolating solution for any type $\mathrm{C}$ solution with only three-form flux

- starting from point $\mathrm{B}$, we can add a constant $B$ field and, using the isometries of the background, T-dualize along $\mathbb{T}^{2}$.

The generic solution in this family has a constant warp factor for large $r$. With a suitable scaling limit we can obtain near-branes solutions whose interpretation will be discussed in the following. Before entering into the details, we discuss some general features of interpolating solutions with vanishing axion.

As already discussed in section 1.1, the most general solution with $\pi / 2<w<\pi$ has a conformally balanced metric ( $J^{2}$ is conformally closed), the three forms are given by

$$
\begin{aligned}
H & =-\mathrm{d}(\cot w J), \\
F_{3} & =-\frac{i}{\sin w} e^{-2 A}(\partial-\bar{\partial})\left(e^{2 A-\phi} J\right)+i \sin w e^{-\phi}(\partial-\bar{\partial})(2 A) \wedge J,
\end{aligned}
$$

and the Bianchi identity for $H$ trivially follows from (4.1a).

If we also choose to have constant axion, the supersymmetry equations in section 1.2 simplify and, most notably, from (1.19a) and (1.18a) we have

$$
\begin{aligned}
\mathrm{d}\left(e^{-\phi} \cos w\right) & =0, \\
\mathrm{~d}\left(e^{2 A-\phi} \sin w\right) & =0,
\end{aligned}
$$

respectively. These equations can be integrated to

$$
\cos w=-e^{\phi-\phi_{\infty}} \tanh \beta, \quad \sin w=\frac{e^{-\phi_{\infty}}}{\cosh \beta} e^{-(2 A-\phi)},
$$

and, as consequence, the warp factor reads

$$
e^{-4 A}=1+\cosh ^{2} \beta\left(e^{-2\left(\phi-\phi_{\infty}\right)}-1\right) .
$$

To simplify comparisons with the existing literature, we used for the integration constants the parametrisation suggested in [9]. $\phi_{\infty}$ is the asymptotic value of the dilaton and $\beta$ is 
the boost parameter entering the chain of dualities in [9]. Notice that we normalized the asymptotic value of the warp factor to one; a generic value can be easily reintroduced.

Finally, a generic feature of interpolating solutions, which follows straightforwardly from the supersymmetry equations, is that the $\mathrm{RR}$ three-form is always proportional to the Hodge star of the NS one

$$
F_{3}=-\frac{e^{-\phi}}{\cos w} * H
$$

This relation generalises the imaginary self-duality condition of type B solutions.

\subsection{The chain of "external" dualities}

It was shown in [9] that, starting from a type $\mathrm{C}$ solution with only three-form flux, it is possible to generated a family of backgrounds of interpolating type satisfying all supersymmetry constraints. All these solutions have zero axion as the original type $\mathrm{C}$ case. More precisely, given any type $\mathrm{C}$ solution with internal metric $\mathrm{d} s_{6 C}^{2}$, fundamental form $J_{C}$ and dilaton $\phi_{C}$, we obtain an interpolating solution of the form

$$
\begin{aligned}
\mathrm{d} s^{2} & =e^{2 A} \mathrm{~d} s_{4}^{2}+e^{-2 A+\phi} \mathrm{d} s_{6 C}^{2} \\
\phi & =\phi_{C}
\end{aligned}
$$

and fluxes

$$
\begin{aligned}
H & =\sinh \beta \mathrm{d}\left(e^{\phi_{C}} J_{C}\right) \\
F_{3} & =e^{\phi_{\infty}} \cosh \beta e^{-2 \phi_{C}} * \mathrm{~d}\left(e^{\phi_{C}} J_{C}\right), \\
F_{5} & =-\frac{i}{2} \sinh 2 \beta e^{\phi_{\infty}}(\partial-\bar{\partial})\left(\phi_{C}\right) \wedge J_{C}^{2} .
\end{aligned}
$$

In [9] this family was derived from the type $\mathrm{C}$ solution via a chain of dualities involving a lift to $\mathrm{M}$ theory and an eleven-dimensional boost. It is straightforward to verify that the above expressions satisfy all supersymmetry constraints.

We can turn to the Bianchi identities now. The closure $H$ is satisfied trivially. A remarkable feature of this solution is that the Bianchi identities for $F_{3}$ and $F_{5}$ reduce to those for the type $\mathrm{C}$ solution ${ }^{6}$

$$
\begin{aligned}
\mathrm{d} F_{3}=0 & \Rightarrow \partial \bar{\partial}\left(e^{-2 A_{C}} J_{C}\right)=0, \\
\mathrm{~d} F_{5}-H \wedge F_{3}=0 & \Rightarrow \partial \bar{\partial}\left(e^{-2 A_{C}} J_{C}\right) \wedge J_{C}=0 .
\end{aligned}
$$

Notice from (4.7) that the new $F_{3}$ is proportional to the type $\mathrm{C}$ one given by (2.3).

In [9], this construction was used to recover the baryonic branch solution of [6] from the pure type $\mathrm{C}$ solution of [11]. However this construction is more general and can be applied to our torsional backgrounds as well.

\footnotetext{
${ }^{6}$ In deriving (4.8b) it is useful to use the primitivity of the form $e^{2 A-\phi} J$ (and in particular that $\partial\left(e^{2 A-\phi} J\right) \wedge \bar{\partial}\left(e^{2 A-\phi} J\right)=-e^{2 A-\phi} J \wedge \partial \bar{\partial}\left(e^{2 A-\phi} J\right)$. Note that since $e^{-2 A_{C}} J_{C}$ is not primitive, the solution of $(4.8 \mathrm{~b})$ requires $\partial \bar{\partial}\left(e^{-2 A_{C}} J_{C}\right)=0$. Remember also that, in type $\mathrm{C}, 2 A_{C}=\phi_{C}$.
} 
The interpolating solutions for our Taub-NUT type C backgrounds are obtained from the above equations with the metric and fundamental forms defined in section 2 . In particular

$$
J_{C}=e^{-\phi_{C}} J_{\mathcal{B}}+e^{\phi_{C}} \frac{i}{2} \Theta \wedge \bar{\Theta} .
$$

Finally, the pure spinors are given by (in terms of the type $\mathrm{C}$ dilaton $\phi=\phi_{C}$ ):

$$
\begin{aligned}
& \left.\Psi_{+}\right|_{\text {int }}=i e^{-\phi_{\infty}} \frac{i e^{-2 A}-\sinh \beta}{\cosh \beta} \exp \left[-\left(\sinh \beta+i e^{-2 A}\right)\left(J_{\mathcal{B}}+\frac{i}{2} e^{2 \phi} \Theta \wedge \bar{\Theta}\right)\right], \\
& \left.\Psi_{-}\right|_{\text {int }}=i e^{-3 A} \omega_{\mathcal{B}} \wedge \Theta .
\end{aligned}
$$

The relation between the dilaton and the warp factor is given by (4.4).

\subsection{The "internal" dualities}

The same solution can be obtained from the type B background of section 3 by a particular $O(2,2)$ transformation consisting of adding a closed $B$ field and T-dualizing along $\mathbb{T}^{2}$.

Let us start from a type B solution with arbitrary parameters, including the volume of $\mathbb{T}^{2}$, which was previously set to one for simplicity. The solution is

$$
\begin{aligned}
\mathrm{d} s_{6}^{2} & =e^{-2 A_{B}}\left(\mathrm{~d} s_{\mathcal{B}}^{2}+R^{2} \mathrm{~d} \theta \otimes \mathrm{d} \bar{\theta}\right), \\
e^{\phi} & =g_{s} \\
B & =B^{1} \wedge \mathrm{d} \theta^{1}+B^{2} \wedge \mathrm{d} \theta^{2},
\end{aligned}
$$

where $A_{B}$ is a solution of the tadpole equation (3.2)

$$
\square_{\mathcal{B}}\left(e^{-4 A_{B}}\right)=\frac{1}{R^{2}} *(F \wedge \bar{F}),
$$

where the rescaling of the $\theta^{I}$ has been taken into account.

We may perform now a constant $B$-transform, an operation that while shifting the $B$-field will not change the flux $H$

$$
B \longrightarrow B+b \mathrm{~d} \theta_{1} \wedge \mathrm{d} \theta_{2}+d J_{\mathcal{B}} .
$$

The crucial term in this redefinition is the constant two-form along the torus, which does not commute with double T-duality. The base-dependent part in the $B$-transform proportional to $J_{\mathcal{B}}$ has been included in order to be able to collect the result of T-duality onto the form (4.10).

A standard application of T-duality along $\theta^{1}$ and $\theta^{2}$ now gives the interpolating solution. In the previous subsection, various arbitrary parameters, including the radius of the torus and the asymptotic value of the warp factor, have been set to one. In order to recover the same normalizations we need to choose $g_{s}=R, d=-b / R^{2}$ and the asymptotic value of $e^{-4 A_{B}}$ equal to one. Obviously, we could relax these conditions and obtain interpolating solutions with arbitrary parameters. Note that the warp factor for the interpolating solution is the same as in type $\mathrm{B}\left(A=A_{B}\right)$ while the dilaton is given by the standard rules of T-duality

$$
e^{-2 \phi}=\frac{1}{g_{s}^{2}}\left(b^{2}+R^{4} e^{-4 A}\right)=\frac{1}{R^{2}}\left(b^{2}+R^{4} e^{-4 A}\right)
$$


from which, by comparison with (4.4), we read the value of the parameters $\beta$ and $\phi_{\infty}$

$$
e^{-2 \phi_{\infty}}=\frac{b^{2}+R^{4}}{R^{2}} \quad \sinh \beta=-\frac{b}{R^{2}} .
$$

Since $e^{-2 \phi}$ and $e^{-4 A}$ are linearly related, they both satisfy the standard tadpole equation. We can choose $\phi=\phi_{C}$ of the form (2.19) for the general Taub-NUT solutions and (2.28) for the Eguchi-Hanson case. $e^{-4 A}$ has a similar form with different coefficients; in particular, for us, its asymptotic value is normalized to one.

There exists another $O(2,2)$ transformation that relates the type B background with the interpolation (4.10). We could start with the type B solution with general parameters and perform a Lunin-Maldacena transformation (LM) [19]

$$
\left(\begin{array}{ll}
1 & 0 \\
\gamma & 1
\end{array}\right) \in \mathrm{SL}(2, \mathbb{Z})
$$

acting on the complexified Kähler parameter of the two-torus. It is easy to check that we obtain again the interpolating solution up to a constant shift of the final $B$ field. The continuous parameter $\gamma$ plays the role of $b$ in the T-duality transformation. The two parameters $\phi_{\infty}$ and $\beta$ are related by a redefinition to the torus radius $R$ in the type $\mathrm{B}$ solution and the value of $\gamma$.

Alternatively, one may start with a D3 branes transverse to $\mathcal{B} \times \mathbb{T}^{2}$ without three form fluxes, and combine a non-constant $B$ transform with twisting of the two-torus in such a way that the original integrable complex structure does not change. The transformed even pure spinor $\Psi_{+}$will generate the correct three-form fluxes. In [20] such a transformation was used to relate compact type $\mathrm{B}$ and $\mathrm{C}$ backgrounds. In our case the novelty is that the the phase of the pure spinor does not change in a discrete fashion.

\subsection{Properties of the interpolating solution}

In this section we will discuss some features of the interpolating solutions described above. For simplicity, we will focus on the simple case of the Eguchi-Hanson base.

The ten-dimensional metric becomes

$$
\mathrm{d} s^{2}=e^{2 A} \mathrm{~d} s_{4}^{2}+m e^{-2 A}\left(\mathrm{~d} s_{\mathcal{B}}^{2}+e^{2 \phi_{C}} \Theta \otimes \bar{\Theta}\right),
$$

with dilaton $\left(\phi=\phi_{C}\right)$ and warp factor

$$
\begin{aligned}
e^{-2 \phi_{C}} & =\frac{|c|^{2}}{a^{4} \sqrt{a^{4}+r^{4}}}+e^{-2 \phi_{\infty}}, \\
e^{-4 A} & =1+\cosh ^{2} \beta\left(e^{-2\left(\phi_{C}-\phi_{\infty}\right)}-1\right) .
\end{aligned}
$$

The fluxes are

$$
\begin{aligned}
F_{3} & =i m e^{\phi_{\infty}} \cosh \beta(\partial-\bar{\partial})\left(e^{-2 \phi_{C}} J_{\mathcal{B}}+\frac{i}{2} \Theta \wedge \bar{\Theta}\right), \\
H & =\frac{i m}{2} \sinh \beta \mathrm{d}\left(e^{2 \phi_{C}} \Theta \wedge \bar{\Theta}\right), \\
F_{5} & =\frac{1}{2} m^{2} \sinh 2 \beta e^{\phi_{\infty}}(\partial-\bar{\partial})\left(\phi_{C}\right) \wedge J_{\mathcal{B}} \wedge \Theta \wedge \bar{\Theta} .
\end{aligned}
$$

We introduced an arbitrary constant $m$ which will be useful very soon. 
For generic $\beta$, the warp factor $A$ goes to a constant for large $r$, as seen from (4.18). As noticed in [9], we can also obtain a near-brane solution by sending $\beta \rightarrow \infty .^{7}$ In order to have a smooth limit, we need to rescale the space-time coordinates and the parameter $m$,

$$
d s_{4}^{2} \rightarrow \frac{|c| e^{\phi_{\infty}}}{a^{3}} \cosh \beta d s_{4}^{2}, \quad m \rightarrow \frac{a^{3}}{|c| e^{\phi_{\infty}} \cosh \beta} .
$$

Factors of $c, a$ and $e^{\phi_{\infty}}$ are introduced for future convenience. In this way, we obtain

$$
\begin{aligned}
\mathrm{d} s^{2} & =e^{2 A} \mathrm{~d} s_{4}^{2}+e^{-2 A}\left(\mathrm{~d} s_{\mathcal{B}}^{2}+e^{2 \phi_{C}} \Theta \otimes \bar{\Theta}\right), \\
e^{-2 \phi_{C}} & =\frac{|c|^{2}}{a^{4} \sqrt{a^{4}+r^{4}}}+e^{-2 \phi_{\infty}}, \\
e^{-4 A} & =\frac{a^{2}}{\sqrt{a^{4}+r^{4}}}, \\
F_{3} & =i \frac{a^{3}}{|c|}(\partial-\bar{\partial})\left(e^{-2 \phi_{C}} J_{\mathcal{B}}+\frac{i}{2} \Theta \wedge \bar{\Theta}\right), \\
H & =i \frac{a^{3}}{2|c|} e^{-\phi_{\infty}} \mathrm{d}\left(e^{2 \phi_{C}} \Theta \wedge \bar{\Theta}\right), \\
F_{5} & =\frac{a^{6}}{|c|^{2}} e^{-\phi_{\infty}}(\partial-\bar{\partial}) \phi_{C} \wedge J_{\mathcal{B}} \wedge \Theta \wedge \bar{\Theta} .
\end{aligned}
$$

Differently from section 2, where we obtained a near-brane solution by sending the asymptotic value of the dilaton to zero, here $\phi_{\infty}$ has an arbitrary value and the near-brane limit is obtained by sending $\beta$ to infinity.

Notice first that the solution is still of interpolating type and is a mixture of those discussed in sections 2 and 3. For large $r$ the metric behaves as

$$
\mathrm{d} s^{2} \sim r \mathrm{~d} s_{4}^{2}+\frac{1}{r}\left(\mathrm{~d} s_{\mathcal{B}}^{2}+e^{2 \phi_{\infty}} \mathrm{d} \theta \otimes \mathrm{d} \bar{\theta}\right)
$$

and there is a non-vanishing $B$ field along the two-torus. The asymptotic solution corresponds to a D5 brane with a non commutative parameter on the world-volume $[17,18]$. The $F_{3}$ flux is supported on the three-sphere at infinity of the ALE space. The $B$ field induces a non trivial D3 charge and the metric looks like that of D3 branes smeared on the two-torus. The torus shrinks for large $r$ but the metric is regular. For small $r$, as in the type $\mathrm{C}$ solution, the internal manifold becomes topologically $\mathbb{R}^{2} \times S^{1} \times S^{3}$, the D3 brane charge vanishes and the $F_{3}$ flux is supported by the IR three-sphere. Notice that the dilaton is running but approaches a constant both for large and small $r$. All this is reminiscent of the gravity dual of the baryonic branch of the Klebanov-Strassler theory [6].

However, differently from the baryonic branch solution, the gauge theory dual to our interpolating solution is less understood. We can however, try to give an interpretation of the parameters in our solution. As discussed in section 2.2, the type $\mathrm{C}$ solution corresponds to a five-brane wrapping a two-torus near an ALE space. The solution is holographically

\footnotetext{
${ }^{7}$ This observation was used to obtain the the gravity dual of the baryonic branch of the Klebanov-Strassler theory [6] (which is a near-brane solution) starting from the generalization of the Maldacena-Nunez solution given in [11].
} 
dual to a little string theory associated to a $\mathbb{C}^{2} / Z_{2}$ singularity. At low energy it reduces to a six-dimensional gauge theory coupled to tensor multiplets with eight supercharges, two gauge groups and two bi-fundamental hyper-multiplets. There are several explicit parameters in the solution which have an interpretation in the dual field theory. $a$ corresponds to a resolution of the singularity and it is usually associated with a baryonic VEV in the gauge theory. $c$ corresponds to a twist of the theory which preserves $\mathcal{N}=2$ supersymmetry. In the interpolating solution, a non-commutative parameter is turned on. As a result the asymptotic value of the dilaton $e^{-2 \phi_{\infty}}$ is finite, setting the difference between the non-commutative and the little string scale.

In the case of the baryonic branch solution [6], the resulting solution is de facto an interpolation between type $\mathrm{C}$ and type $\mathrm{B}$ solutions. Indeed, with a suitable tuning of the parameters corresponding to the baryonic VEV and the gauge coupling constant, the solution connects the Klebanov-Strassler point (type B) with the Maldacena-Nunez solution (type C). Interestingly, a similar phenomenon happens for our solutions; by varying parameters, we can interpolate between the near-brane type $\mathrm{B}$ solution of section 3 and the near-brane type C solution of section 2 . We can conveniently use $\phi_{\infty}$ and the value $\phi_{0}$ of the dilaton at $r=0$ as parameters. We will keep $\phi_{0}$ and $a$ fixed and we will vary $\phi_{\infty}$. We have a consistent solution for all $\phi_{\infty} \geq \phi_{0}$. $c$ is then determined as

$$
|c|=a^{3} \sqrt{e^{-2 \phi_{0}}-e^{-2 \phi_{\infty}}} .
$$

For $\phi_{\infty} \rightarrow \phi_{0}$, we have that $c \rightarrow 0$ and the metric becomes a direct product for all values of $r^{8}$

$$
\begin{aligned}
\mathrm{d} s^{2} & =e^{2 A} d s_{4}^{2}+m e^{-2 A}\left(\mathrm{~d} s_{\mathcal{B}}^{2}+e^{2 \phi_{0}} \mathrm{~d} \theta \otimes \mathrm{d} \bar{\theta}\right), \\
e^{-4 A} & =\frac{a^{2}}{\sqrt{a^{4}+r^{4}}} .
\end{aligned}
$$

This is the same as the B solution discussed in section 3. A short calculation shows that, in the limit $c \rightarrow 0$, also the fluxes are consistent with a type B solution with constant dilaton. On the other hand, for $\phi_{\infty} \rightarrow \infty, 2 A \sim \phi_{C}$, the $H$ and $F_{5}$ fluxes vanish and we recover the type $\mathrm{C}$ solution of section 2 with an effective value of the parameter $c=a^{3} e^{-\phi_{0}}$. All metrics in the interpolation have the same asymptotic behavior. At the endpoint $\phi_{\infty} \rightarrow \infty$ the dilaton blows up at infinity

$$
e^{-2 \phi} \sim \frac{1}{\sqrt{a^{4}+r^{4}}}
$$

From the B point perspective we have turned on a twist $c$ and simultaneously changed the value of $e^{-2 \phi_{\infty}}$ which controls the gauge coupling of the dual theory. When $e^{-2 \phi_{\infty}} \rightarrow 0$ the asymptotic behavior of the solution changes. This is similar to what happens for the baryonic branch solution and the interpolation between the Klebanov-Strassler and the Maldacena-Nunez solution [6].

\footnotetext{
${ }^{8}$ Without factors of $c$ in the rescaling (4.20), we would have obtained a type B case by sending $c \rightarrow 0$ without varying $\phi_{\infty}$. However equations (4.21) imply a constant warp factor $A$ and so an uninteresting Calabi-Yau solution consisting of an ALE space times a two-torus and no flux.
} 


\section{$5 \quad$ Discussion and conclusions}

In this paper we constructed new interpolating solution of type IIB based on non compact fibration of a two-torus over a Taub-NUT space.

In the $\mathcal{N}=2$ case the resulting family of solution is regular. We discussed in great details the case of the Eguchi-Hanson base where the solution and its topology can be studied quite explicitly. In the general solution, the asymptotic value of the warp factor is constant, corresponding to an asymptotically unwarped background, but we also considered a near-brane limit. The backgrounds depend on many parameters, including the asymptotic values of the dilaton and warp factor, and the details of the fibration. Some of these parameters will be quantized. This is certainly the case for the Chern class of the fibration, c. In addition, since we have non-trivial three cycles in the geometry, the periods of the NS and RR three-forms lead to other quantization conditions.

Since our solutions are non compact, the question of a possible gauge dual arises naturally. Let us consider first the simple case of the type $\mathrm{C}$ solution, corresponding to a five-brane wrapped on a two-torus near an ALE space. The dual gauge theory should come from the compactification on the torus of the six-dimensional gauge theory living on a D5-brane near an ALE space. When the tours is replaced by flat space $(c=0)$ and $a=0$, the solution is the holographic dual of a little string theory associated to a $\mathbb{C}^{2} / Z_{2}$ singularity. At low energy it reduces to a six-dimensional gauge theory coupled to tensor multiplets with eight supercharges, two gauge groups and two bi-fundamental hyper-multiplets. In the case of a more general ALE space with $N$ centers, the theory is based on an affine $A_{N}$ quiver coupled to tensor multiplets, with $N$ gauge groups and bi-fundamental hyper-multiplets connecting neighbouring groups [21]. In this picture, the parameter $a$ corresponds to a resolution of the singularity and it is usually associated with a baryonic VEV in the gauge theory. The parameter $c$ is less straightforward to interpret and is related to the compactification of the theory on the torus. It should correspond to a twist of the theory by some global $\mathrm{U}(1)$ in such a way to preserve $\mathcal{N}=2$ supersymmetry.

In the interpolating solution, a non-commutative parameter is also turned on.

What is interesting and unusual is that, in spite of being $\mathcal{N}=2$, the dual of such gauge theory is regular and, in the IR, is topologically similar to the known IR behaviour of $\mathcal{N}=1$ confining theories with a non-trivial three-sphere that supports the RR flux. In contrast with the MN and KS case, there is an extra circle, surviving the twist, where we could wrap strings. It would be interesting to understand the role of such strings in the IR dynamics and to understand better the structure and moduli space of the $\mathcal{N}=2$ dual theory. We will leave these issues for future work.

\section{Acknowledgments}

We would like to thank I. Bena, S. Giusto, L. S. Tseng and N. Warner for useful discussions. We would also like to thank KITP, UC Santa Barbara (RM) and GGI Center of Physics in Florence (MP and AZ) for hospitality during the course of this work. This work is supported in part by RTN contracts MRTN-CT-2004-005104 and MRTN-CT-2004-512194 and by ANR grants BLAN05-0079-01 (DA and MP) and BLAN06-3-137168 (RM). A. Z. is supported in part by INFN and MIUR under contract 2007-5ATT78-002. 
Open Access. This article is distributed under the terms of the Creative Commons Attribution Noncommercial License which permits any noncommercial use, distribution, and reproduction in any medium, provided the original author(s) and source are credited.

\section{References}

[1] M. Graña, R. Minasian, M. Petrini and A. Tomasiello, Supersymmetric backgrounds from generalized Calabi-Yau manifolds, JHEP 08 (2004) 046 [hep-th/0406137] [SPIRES].

[2] M. Graña, R. Minasian, M. Petrini and A. Tomasiello, Generalized structures of $N=1$ vacua, JHEP 11 (2005) 020 [hep-th/0505212] [SPIRES].

[3] M. Graña and J. Polchinski, Gauge/gravity duals with holomorphic dilaton, Phys. Rev. D 65 (2002) 126005 [hep-th/0106014] [SPIRES].

[4] I.R. Klebanov and M.J. Strassler, Supergravity and a confining gauge theory: Duality cascades and $\chi$ SB-resolution of naked singularities, JHEP 08 (2000) 052 [hep-th/0007191] [SPIRES];

[5] J.M. Maldacena and C. Núñez, Towards the large- $N$ limit of pure $N=1$ super Yang-Mills, Phys. Rev. Lett. 86 (2001) 588 [hep-th/0008001] [SPIRES].

[6] A. Butti, M. Graña, R. Minasian, M. Petrini and A. Zaffaroni, The baryonic branch of Klebanov-Strassler solution: A supersymmetric family of $\mathrm{SU}(3)$ structure backgrounds, JHEP 03 (2005) 069 [hep-th/0412187] [SPIRES].

[7] E. Goldstein and S. Prokushkin, Geometric model for complex non-Kähler manifolds with SU(3) structure, Commun. Math. Phys. 251 (2004) 65 [hep-th/0212307] [SPIRES].

[8] J.-X. Fu, L.-S. Tseng and S.-T. Yau, Local Heterotic Torsional Models, Commun. Math. Phys. 289 (2009) 1151 [arXiv:0806.2392] [SPIRES].

[9] J. Maldacena and D. Martelli, The unwarped, resolved, deformed conifold: fivebranes and the baryonic branch of the Klebanov-Strassler theory, JHEP 01 (2010) 104 [arXiv:0906.0591] [SPIRES].

[10] I. Bena, N. Bobev and N.P. Warner, Spectral Flow and the Spectrum of Multi-Center Solutions, Phys. Rev. D 77 (2008) 125025 [arXiv:0803.1203] [SPIRES].

[11] R. Casero, C. Núñez and A. Paredes, Towards the string dual of $N=1$ SQCD-like theories, Phys. Rev. D 73 (2006) 086005 [hep-th/0602027] [SPIRES].

[12] O. Aharony, M. Berkooz, D. Kutasov and N. Seiberg, Linear dilatons, NS5-branes and holography, JHEP 10 (1998) 004 [hep-th/9808149] [SPIRES].

[13] P. Bouwknegt, J. Evslin and V. Mathai, T-duality: Topology change from H-flux, Commun. Math. Phys. 249 (2004) 383 [hep-th/0306062] [SPIRES].

[14] M. Graña, R. Minasian, M. Petrini and D. Waldram, T-duality, Generalized Geometry and Non-Geometric Backgrounds, JHEP 04 (2009) 075 [arXiv: 0807.4527] [SPIRES].

[15] M. Bertolini et al., Supersymmetric 3-branes on smooth ALE manifolds with flux, Nucl. Phys. B 617 (2001) 3 [hep-th/0106186] [SPIRES].

[16] A.R. Frey and M. Graña, Type IIB solutions with interpolating supersymmetries, Phys. Rev. D 68 (2003) 106002 [hep-th/0307142] [SPIRES]. 
[17] A. Hashimoto and N. Itzhaki, Non-commutative Yang-Mills and the AdS/CFT correspondence, Phys. Lett. B 465 (1999) 142 [hep-th/9907166] [SPIRES].

[18] J.M. Maldacena and J.G. Russo, Large-N limit of non-commutative gauge theories, JHEP 09 (1999) 025 [hep-th/9908134] [SPIRES].

[19] O. Lunin and J.M. Maldacena, Deforming field theories with $\mathrm{U}(1) \times \mathrm{U}(1)$ global symmetry and their gravity duals, JHEP 05 (2005) 033 [hep-th/0502086] [SPIRES].

[20] D. Andriot, R. Minasian and M. Petrini, Flux backgrounds from Twists, JHEP 12 (2009) 028 [arXiv: 0903.0633] [SPIRES].

[21] K.A. Intriligator, New string theories in six dimensions via branes at orbifold singularities, Adv. Theor. Math. Phys. 1 (1998) 271 [hep-th/9708117] [SPIRES]. 